\title{
SEMILATTICE CONGRUENCES VIEWED FROM QUASI-ORDERS
}

\author{
TAKAYYUKI TAMURA
}

\begin{abstract}
Let $S$ be a semigroup. This paper proves that the smallest semilattice congruence $\rho_{0}$ containing a compatible binary relation $\xi$ on $S$ equals the natural equivalence of the smallest lowerpotent positive quasi-order $\sigma_{0}$ containing $\xi$.
\end{abstract}

1. Introduction. Recently the greatest semilattice decomposition of semigroups has been studied by Putcha [1] and the author [5], [6]. It is noted in [1], [5] that the smallest semilattice congruence can be obtained in the natural way as a generalization of the concept of "archimedeaness". On the other hand it is constructed by quasi-orders: the author proved in [6] that the smallest semilattice congruence is obtained from the smallest power-potent, positive half-congruence; Putcha [1] proved that the smallest semilattice congruence equals the smallest equivalence satisfying certain identities. This paper generalizes the above result in [6], that is, the condition "half-congruence" can be replaced by "quasi-order". The attainability of the system of identities $\left\{x=x^{2}\right.$, $x y=y x\}$ can be proved in a simpler way than before [2], [3], [5], [6].

2. Preliminaries. All binary relations considered here are assumed to be nonempty. In this paper, "equivalence" is the synonym to "equivalence relation" and "congruence" is that to "congruence relation". A quasiorder is defined to be a reflexive transitive relation. Let $S$ be a semigroup. A relation $\xi$ on $S$ is called right (left) compatible if $a \xi b$ implies $a c \xi b c$ $(c a \xi c b)$ for all $c \in S$; $\xi$ is called compatible if it is right and left compatible. A compatible quasi-order is called a half-congruence. A relation $\xi$ on $S$ is called lower-potent if $a^{2} \xi a$ for all $a \in S$. $\xi$ is called positive if $a \xi a b$ and $a \xi b a$ for all $a, b \in S$. If $\xi$ is a quasi-order on a set $S, \xi^{-1}$ is defined by $\xi^{-1}=\{(y, x):(x, y) \in \xi\}$. Let $\xi=\xi \cap \xi^{-1}$. Then $\xi \neq \varnothing$ and $\tilde{\xi}$ is an equivalence on $S$. $\tilde{\xi}$ is called the natural equivalence of a quasi-order $\xi$. If $S$ is a semigroup and if $\xi$ is a half-congruence, $\xi$ is a congruence on $S$.

Received by the editors August 14, 1972 and, in revised form, January 18, 1973.

AMS (MOS) subject classifications (1970). Primary 20M99.

Key words and phrases (Smallest) semilattice congruence, quasi-orders, lower-potent, positive, half-congruences, compatible, natural equivalences, natural quasi-orders, attainability of semilattice.

(c) American Mathematical Society 1973 
Let $\rho$ be a semilattice congruence on a semigroup $S$, i.e., a congruence $\rho$ on $S$ such that $S / \rho$ is a semilattice. Let $f: S \rightarrow S / \rho$ be the natural homomorphism. Define a relation $\eta_{\rho}$ on $S$ by

$$
a \eta_{\rho} b \text { if and only if } f(a) f(b)=f(b) \text {. }
$$

Then $\eta_{\rho}$ is a lower-potent positive half-congruence on $S$ and $\rho=\eta_{\rho} \cap \eta_{\rho}^{-1}$. $\eta_{\rho}$ is called the natural quasi-order of a semilattice congruence $\rho$. The author proved in [6] the following:

THEOREM 2.1. Let $\sigma$ be a lower-potent positive half-congruence on a semigroup $S$. Then the natural equivalence $\tilde{\sigma}$ of $\sigma$ is a semilattice congruence on $S$. Every semilattice congruence on $S$ is obtained in this manner, and the correspondence $\sigma \rightarrow \tilde{\sigma}$ is one-to-one and inclusion preserving.

COROLlaRY 2.2. The smallest semilattice congruence on $S$ equals the natural equivalence $\tilde{\sigma}_{0}$ of the smallest lower-potent positive half-congruence $\sigma_{0}$ on $S$.

In this paper we generalize Corollary 2.2 so that we can remove compatibility from the assumption on $\sigma_{0}$ in Corollary 2.2.

THEOREM 2.3. For any compatible binary relation $\xi$ on $S$, the smallest semilattice congruence $\rho_{0}$ containing $\xi$ equals the natural equivalence of the smallest lower-potent positive quasi-order $\sigma_{0}$ containing $\xi$.

COROLlary 2.4. The smallest semilattice congruence on $S$ equals the natural equivalence of the smallest lower-potent positive quasi-order on $S$.

3. Proof of Theorem 2.3. In (3.1) through (3.4) below we assume that $\sigma$ is a lower-potent positive quasi-order containing $\xi$.

$$
a b \tilde{\sigma} a b a \tilde{\sigma} b a b \tilde{\sigma} b a \text { for all } a, b \in S \text {. }
$$

Proof. By using the assumption, $a b \sigma a b a \sigma b a b a=(b a)^{2} \sigma b a$, hence $a b \sigma a b a \sigma b a$. Likewise $b a \sigma b a b \sigma a b$. Combining these we get the conclusion (3.1).

$$
\text { xay } \tilde{\sigma} x a^{2} y \text { for all } a \in S \text {, all } x, y \in S^{1}{ }^{1}
$$

Proof. By positiveness and (3.1) we have xay $\sigma$ ayx $\sigma$ ayxa $\sigma x a^{2} y$ and $x a^{2} y \sigma$ ayxa $\sigma$ ayxayx $\sigma$ ayx $\sigma$ xay.

$$
a b \sigma a c b \text { for all } a, b, c \in S \text {. }
$$

Proof. By positiveness and (3.1) we have $a b \sigma b a \sigma c b a \sigma a c b$. xaby $\tilde{\sigma} x b a y$ for all $a, b \in S$, all $x, y \in S^{1}$.

\footnotetext{
${ }^{1} S^{1}=S \cup\{1\}$. (3.2) means " $x a y \tilde{\sigma} x a^{2} y$, ay $\tilde{\sigma} a^{2} y, x a \tilde{\sigma} x a^{2}, a \tilde{\sigma} a^{2}$ for all $a \in S$,
} all $x, y \in S . "$ 
Proof. By (3.3) and (3.2) we have $x a b y \sigma x b a b y \sigma x b a b a y=x(b a)^{2} y \sigma$ $x b a y$ and hence $x a b y \sigma x b a y$. Likewise $x b a y \sigma x a b y$.

(3.5) [4] The smallest semilattice congruence $\rho_{0}$ containing a compatible relation $\xi$ equals the symmetric transitive closure of

$$
\begin{aligned}
A= & \xi \cup\{(x, x): x \in S\} \cup\left\{\left(x a^{2} y, x a y\right): a \in S, x, y \in S^{1}\right\} \\
& \cup\left\{(x a b y, x b a y): a, b \in S, x, y \in S^{1}\right\} .
\end{aligned}
$$

Now, it follows from (3.2) and (3.4) that $\rho_{0} \subseteq \tilde{\sigma}$ for all lower-potent positive quasi-orders $\sigma$ containing $\xi$, hence $\rho_{0} \subseteq \tilde{\sigma}_{0}$. On the other hand let $\eta_{\rho_{0}}$ be the natural quasi-order of $\rho_{0}$. Since $\eta_{\rho_{0}}$ is a lower-potent positive quasi-order containing $\xi$, we have $\sigma_{0} \subseteq \eta_{\rho_{0}}$ which implies $\sigma_{0}^{-1} \subseteq \eta_{\rho_{0}}^{-1}$ and so $\tilde{\sigma}_{0}=\sigma_{0} \cap \sigma_{0}^{-1} \subseteq \eta_{\rho_{0}} \cap \eta_{\rho_{0}}^{-1}=\rho_{0}$. Consequently we get $\rho_{0}=\tilde{\sigma}_{0}$. This completes the proof of Theorem 2.3.

Corollary 2.4 is obtained from the case $\xi=\{(x, x): x \in S\}$.

4. Alternate proof of attainability. A semigroup $S$ is called $\mathscr{S}$ indecomposable if only the universal relation $\omega_{S}=S \times S$ is a semilattice congruence on $S$. The decomposition of $S$ induced by the smallest semilattice congruence on $S$ is called the greatest semilattice decomposition of $S$. Clearly $\omega_{S}=\xi \cap \xi^{-1}$ if and only if $\xi=\omega_{S}$. The following theorem was proved in [2], [3], [5], [6], but we give an alternate proof.

THEOREM 4.1. In the greatest semilattice decomposition of a semigroup, each congruence class is an $\mathscr{S}$-indecomposable semigroup.

Proof. Let $S$ be a semigroup and $\sigma_{0}$ the smallest lower-potent positive quasi-order on $S$. Let $S_{\alpha}$ be a $\tilde{\sigma}_{0}$-class of $S$, and $\sigma_{1}$ the smallest lowerpotent positive quasi-order on $S_{\alpha}$. We will prove $\sigma_{1}=\omega_{S_{\alpha}}$ which implies $\tilde{\sigma}_{1}=\omega_{S_{\alpha}}$. Let $p, q \in S_{\alpha}$, i.e., $p \tilde{\sigma}_{0} q$. There is a sequence

$$
p=p_{0}, \quad p_{1}, \cdots, p_{n-1}, \quad p_{n}=q
$$

such that $\left(p_{i}, p_{i+1}\right) \in A$ because of (3.5) $(i=0,1, \cdots, n-1)$. Accordingly $p=p_{0} \sigma_{0} p_{1} \sigma_{0} \cdots \sigma_{0} p_{n-1} \sigma_{0} q \sigma_{0} p$ hence $p \sigma_{0} p_{i}$ and $p_{i} \sigma_{0} p$ for all $i(i=$ $1, \cdots, n-1)$. Thus $p_{i} \in S_{\alpha}(i=1, \cdots, n-1)$. If we prove $A \mid S_{\alpha} \subseteq \sigma_{1}$, then $p_{i} \sigma_{1} p_{i+1}(i=0, \cdots, n-1)$ and so $p \sigma_{1} q$ by transitivity, hence $\sigma_{1}=\omega_{S_{\alpha}}$.

To prove $A \mid S_{\alpha} \subseteq \sigma_{1}$ we prove

(i) $a, a b \in S_{\alpha}$ implies $a \sigma_{1} a b$.

(ii) $a, b a \in S_{\alpha}$ implies $a \sigma_{1} b a$.

(iii) $a \in S_{\alpha}$ implies $a^{2} \sigma_{1} a$.

The last one obviously holds. To see (i), since $a, a b$ and $b a b$ are in $S_{\alpha}$ by (3.1), we have $a \sigma_{1} a(b a b)=(a b)^{2} \sigma_{1} a b$ where we have used the positiveness of $\sigma_{1}$. Hence $a \sigma_{1} a b$. To see (ii), since $a, b a$ and $b a b$ are in $S_{\alpha}$, we get

$$
a \sigma_{1}(b a b) a=(b a)^{2} \sigma_{1} b a .
$$

This completes the proof of Theorem 4.1. 
5. Remark. The natural equivalence of a lower-potent positive quasi-order need not be a semilattice congruence as shown in Example 5.1 below. Accordingly, in Theorem 2.1 we cannot replace "half-congruence" by "quasi-order".

ExAmple 5.1. Let $S$ be a semigroup defined by the Cayley table:

\begin{tabular}{l|llll}
\multicolumn{1}{l}{} & \multicolumn{1}{c}{0} & 1 & 2 & 3 \\
\cline { 2 - 5 } 0 & 0 & 1 & 0 & 0 \\
1 & 1 & 0 & 1 & 1 \\
2 & 0 & 1 & 2 & 0 \\
3 & 0 & 1 & 0 & 3
\end{tabular}

Let $\sigma=\{(0,0),(1,1),(2,2),(3,3),(0,1),(1,0),(2,3),(3,2),(2,0)$, $(2,1),(3,0),(3,1)\} . \sigma$ is a lower-potent positive quasi-order, but

$$
\tilde{\sigma}=\{(0,0),(1,1),(2,2),(3,3),(0,1),(1,0),(2,3),(3,2)\}
$$

is not a semilattice congruence, not even a congruence.

Let $\sigma$ be a quasi-order. In order that $\tilde{\sigma}$ be a semilattice congruence, the lower-potency of $\sigma$ is a necessary condition, but the positiveness of $\sigma$ is not necessary as shown in [6]. It is noted there that the compatibility of $\sigma$ is not a necessary condition.

EXAMPLE 5.2. Let $S$ be the same as in Example 5.1. Let $\sigma=\{(0,0)$, $(1,1),(2,2),(3,3),(0,1),(1,0),(2,0),(2,1),(2,3),(3,0),(3,1)\}$. Then $\sigma$ is a lower-potent positive quasi-order, but neither left nor right compatible since $(2,3) \in \sigma$, but $(0,3) \notin \sigma$. However, $\tilde{\sigma}=\{(0,0),(1,1)$, $(2,2),(3,3),(0,1),(1,0)\}$ is the smallest semilattice congruence on $S$.

Putcha proved in [1] the following:

THEOREM 5.3. The smallest semilattice congruence on $S$ equals the smallest equivalence $\xi_{0}$ containing the relation $x y \equiv x y x \equiv y x$ for all $x, y \in S^{1}$.

If $\sigma$ is a quasi-order and if $\tilde{\sigma}$ is a semilattice congruence, then $\sigma$ satisfies the same condition as that of Theorem 5.3, i.e.,

$$
x y \sigma x y x \sigma y x \text { for all } x, y \in S^{1}
$$

but the converse is not true in general as Example 5.1 shows. Even if $\sigma$ is positive and satisfies (5.3.1), $\sigma$ need not be compatible as Example 5.1 shows. Also a half-congruence which satisfies (5.3.1) need not be positive as shown in [6]. Note that an equivalence which satisfies (5.3.1) is not necessarily a semilattice congruence as Example 5.1 shows.

What is a necessary and sufficient condition on $\sigma$ in order that $\tilde{\sigma}$ be a semilattice congruence? 
Proposition 5.4. Let $\sigma$ be a quasi-order on a semigroup $S . \tilde{\sigma}$ is a semilattice congruence on $S$ if and only if $\sigma$ satisfies

(5.4.1) $a^{2} \sigma$ a for all $a \in S$,

(5.4.2) $a \sigma a^{2}$ for all $a \in S$,

(5.4.3) $a b \sigma b a$ for all $a, b \in S$,

(5.4.4) $a \sigma b$ and $b \sigma$ implies ac $\sigma b c$ for all $a, b, c \in S$.

This proposition is very obvious, but what is emphasized here is that no one of the four conditions can be excluded.

The fourth condition cannot be derived from the first three conditions as Example 5.1 shows.

The third cannot be derived from the other: an equality relation on any noncommutative idempotent semigroup is an example.

The second cannot be derived from the other:

Let $\{S=1,2,3, \cdots\}$ be the positive integer semigroup with addition. Define $\sigma$ on $S$ by $x \sigma y$ if and only if $x \geqq y$ where $\geqq$ is the usual order. This $\sigma$ is an example which shows this.

The first one cannot be derived from the other: this is shown in the dual case of the preceding example.

Immediately we have

Proposition 5.5. Let $\sigma$ be a lower-potent positive quasi-order on $S$. $\tilde{\sigma}$ is a semilattice congruence on $S$ if and only if $a \sigma b$ and $b \sigma a$ implies $a c \sigma b c$ for all $a, b, c \in S$.

The author is grateful for the referee's suggestion.

\section{BiBLIOGRAPHY}

1. M. S. Putcha, Minimal sequences in semigroups (to appear).

2. T. Tamura, The theory of construction of finite semigroups. I, Osaka Math. J. 8 (1956), 243-261. MR 18, 717.

3. - Another proof of a theorem concerning the greatest semilattice-decomposition of a semigroup, Proc. Japan Acad. 40 (1964), 777-780. MR 31 \#3530.

4. - The theory of operations on binary relations, Trans. Amer. Math. Soc. 120 (1965), 343-358; errata, ibid. 123 (1966), 273. MR 35 \#273.

5. - Note on the greatest semilattice decomposition of a semigroup, Semigroup Forum 4 (1972), 255-261.

6. — Quasi-orders, generalized archimedeaness and semilattice decomposition (to appear).

Department of Mathematics, University of California, Davis, California 95616 